\title{
The effect of Oxygen application with nCPAP for the prevention of desaturation during EBUS-TBNA
}

\author{
Ozge Oral Tapan ${ }^{1}$, Sebahat Genc ${ }^{1}$, Kemal Can Tertemiz ${ }^{2}$, Aylin Ozgen Alpaydin², Oya \\ $\mathrm{Itil}^{3}$, and Leyla Iyilikci Karaoglan ${ }^{2}$ \\ ${ }^{1}$ Mugla Sitki Kocman University \\ ${ }^{2}$ Dokuz Eylül University \\ ${ }^{3}$ Dokuz Eylul University
}

October 12,2020

\begin{abstract}
Background: EBUS-TBNA is a frequently used diagnostic method for mediastinal/hilar lymphadenopathies and masses. This procedure is performed with intravenous sedation (IVS). During IVS, patients often develop hypoxemia and nasal oxygen delivery is insufficient in some patients. The aim of this study was to investigate the effect of oxygen application with nCPAP on hypoxemia during EBUS-TBNA. Methods: Patients with EBUS-TBNA indication who did not have any serious heart-lung disease were randomly divided into two groups. One group received only oxygen and the other group received nCPAP+oxygen. Patient characteristics, arterial oxygen saturations, anesthetic agents, CPAP pressures, oxygen concentrations and processing times were recorded during the procedure. Practitioner satisfaction was evaluated at the end. Results: $29 \mathrm{nCPAP}+$ oxygen, 31 oxygen patients were included in the study. There were no significant differences in terms of age, sex, smoking history and presence of additional diseases in two groups. Neck circumference, BMI and STOP BANG questionnaire values were similar. Desaturation time was significantly longer in oxygen group than nCPAP+oxygen group (316 \pm 390 sec, $12 \pm 118$ sec, respectively, $\mathrm{p}=0,019)$. Snoring was detected during the procedure in 22 patients in the oxygen group and in 11 patients in the nCPAP group $(\mathrm{p}=0,01)$. There were no serious complications in both groups. Practitioner satisfaction was higher in the nCPAP group but this was not statistically significant $(\mathrm{p}=0,052)$. Conclusions: Oxygen application by nCPAP during EBUS-TBNA under IVS, significantly reduces desaturation time. Oxygen delivery with nCPAP seems to be a better choice especially for the patients with high Mallampati index.
\end{abstract}

\section{Introduction}

Endobronchial ultrasound-guided transbronchial needle aspiration (EBUS-TBNA) is a minimally invasive diagnostic method for mediastinal/hilar lymphadenopathies and masses [1]. During the EBUS-TBNA, all relevant lymph node stations should be evaluated and optimal results can be obtained by three aspirations per lymph node station [2]. The procedure induces cough, increases airway secretions, and reduces airway caliber. Because of these reasons, the procedure should be performed with sedation that provides both patient's comfort and bronchoscopist's ability for obtaining adequate tissue. Intravenous sedation (a combination of alfentanyl/fentanyl, propofol, and/or midazolam) is commonly used in EBUS-TBNA procedure. However desaturation is the major problem during sedation because sedation causes upper airway obstruction, respiratory depression and hypoventilation due to muscle relaxation [3,4]. Hypoxemia can lead to sympathetic activation and may cause tachycardia and hypertension. During bronchoscopic procedures, patients stay under spontaneous breathing and Oxygen is delivered through a mask. Arterial Oxygen saturation should be at least $90 \%$ to reduce the risk of significant arrhythmia [5]. Drugs for anesthesia or sedation can increase the severity of obstructive sleep apnea. Propofol is known to contribute to the narrowing of the upper airway by decreasing the activity of genioglossus muscle in proportion to its concentration. [6]. Continuous positive 
airway pressure (CPAP) has a mechanical effect that increases the intraluminal pressure of the upper airway above the positive transmural pressure of the pharynx and hypopharynx. CPAP has been shown to improve the decreased tidal volume and airflow during sedation for bronchoscopy in children [7]. Additionally, jawthrust and CPAP application have been shown to improve ventilation in infants under anesthesia [8]. To our knowledge, there is no study on the effect of nCPAP on Oxygen desaturation during the EBUS-TBNA. In this study, our aim was to investigate the effect of Oxygen application with nCPAP on hypoxemia during EBUS-TBNA.

\section{Materials and Methods}

\section{Ethics}

Ethics Committee approval for our study was obtained from institutional Ethics Committee (Dokuz Eylul University, School of Medicine, research number: 2015-22-02), and written informed consent was obtained from the participants in accordance with the Helsinki declaration.

\section{Study Design}

Patients with mediastinal or hilar lesions that had an indication of EBUS-TBNA were evaluated. Eligible subjects without any serious and acute respiratory and/or cardiovascular disease were included in the study. Other exclusion criteria were the chronic hypoxemia ( $\mathrm{SpO} 290 \%$ at room air), regular use of benzodiazepinerelated drugs and dementia. The study was planned as a randomized controlled trial. Patients were randomly assigned to two groups. Sample size was calculated by G power analysis. When the effect size was taken as 0,5 , $80 \%$ power (1-beta), type 1 error rate (alpha) was calculated as 0,05 , it was decided to take 30 patients in both groups. Group 1 was consisted of EBUS-TBNA procedure with nCPAP+Oxygen (6-14 mBar+4-10 lt/dk) and Group 2 with only Oxygen (4-10 lt/dk). EBUS-TBNA procedures were performed by the same pulmonologist. A flexible convex-probe ultrasonic-puncture bronchoscope with a linear scanning transducer at a frequency of 7,5 MHz (EB-530US, SU-8000 Endoscopic Ultrasound System, Fujifilm, Tokyo, Japan) and Standard 22gauge needle (Medi-Globe, Germany) were used for EBUS-TBNA procedure. The procedure was performed under moderate IVS with the accompaniment of an anesthesiologist. After premedication with midazolam $0,25 \mathrm{mg} / \mathrm{kg}$, fentanil received $1 \mathrm{mcg} / \mathrm{kg}$ infusion in all cases. Two minutes after the start of the remifentanil infusion, propofol was given as $0,5 \mathrm{mg} / \mathrm{kg}$ iv and the Ramsay sedation score was tried to be between 3-4. When additional dose was needed, $0,25 \mathrm{mg} / \mathrm{kg}$ propofol iv was given. Nasal CPAP (System One CPAP, Phillips Respironics, USA) was applied by another pulmonologist. Demographic data, comorbidities, smoking history, neck circumference, body mass index (BMI), and Mallampati indexes of the participants were recorded. All of the patients answered the STOP BANG Questionnaire [9] (Table 1). During the procedure; peripheral Oxygen saturation (SpO2), desaturation time, electrocardiogram, arterial blood pressure, anesthetic agents, sedation level, CPAP pressure, Oxygen concentration, complications, interventions, processing time, and practitioner satisfaction (best score: $5 / 5$ ) were evaluated. After waking up in an average of 5 minutes, all patients sent to the recovery room. They were discharged after being kept under observation for 2 hours.

\section{Statistics}

Statistical analyses were performed using IBM SPSS statistics version 22. Chi-square for categorical variables, T-test or Mann-Whitney U test for numerical variables were used. Spearman Test was used for correlation analysis. A $\mathrm{p}$ value of $<0,05$ was accepted for statistical significance.

\section{Results}

Sixty patients participated in the study. Of the patients, 29 were in nCPAP+Oxygen group and 31 were in the Oxygen group. There was not any serious complication reported during the procedure in both of groups. Of 60 patients, 3 patients had minör hemorrhage. Two of them were in nCPAP+Oxygen group and one was in the Oxygen group. Snoring was detected during the procedure in 22 patients in the Oxygen group while in 11 people in the nCPAP group $(\mathrm{p}=0,01)$. Electrocardiograms and arterial blood pressure levels of all patients were in normal limits. There were no significant differences in terms of age, sex, smoking history and presence of additional diseases in two groups. Neck circumference, BMI and STOP BANG 
questionnaire values were similar. The Mallampati index was significantly higher in the nCPAP+Oxygen group $(\mathrm{p}=0,048)$. Comorbidities were chronic obstructive pulmonary disease (COPD), hypertension and malignancy. The clinical characteristics of the patients are shown in Table 2.

During the EBUS-TBNA procedure; there were no significant differences between the mean values of initial, final and the lowest Oxygen saturations; and propofol, fentanyl and midazolam doses of two groups. The mean procedure time and the practitioner satisfaction were similar in both groups. The mean desaturation time during the procedure was significantly lower in the $\mathrm{nCPAP}+$ Oxygen group $(\mathrm{p}=0,019)$. High Mallampati index was significantly assocciated with the duration of desaturation and the lowest Oxygen saturation ( $\mathrm{p}=0,006, \mathrm{p}=0,006$, respectively) in Oxygen group while there was no association in CPAP+Oxygen group. Data about Oxygen saturation and the anesthetic drugs are shown in Table 3.

Oxygen saturation decreased to below $80 \%$ in 19 patients (61\%) of the oxygen group (Figure 1), while in 10 patients $(34,5 \%)$ of the nCPAP+oxygen group (Figure 2$)(\mathrm{p}=0,034)$.

\section{Discussion}

In this study we evaluated the benefits of nCPAP use for Oxygenation during EBUS-TBNA. Several studies about the effectiveness of noninvasive ventilation (NIV) in improving oxygenation during bronchoscopy in intensive care units have been reported. Antonelli $\mathrm{M}$ et al. [10] found that noninvasive positive-pressure ventilation (NPPV) that was delivered through a full-face mask was superior to Oxygen supplementation alone in improving gas exchange during and after diagnostic bronchoscopy in patients with severe hypoxemia. According to Murgu et al. [11] NPPV was an alternative to endotracheal intubation for flexible bronchoscopy in many patients with severe refractory hypoxemia, severe COPD, postoperative respiratory failure, severe obstructive sleep apnea and obesity hypoventilation syndrome. Our patients did not have any hypoxemia, and we applied Oxygen with nasal CPAP mask under sedation. In our study, all patients tolerated EBUS-TBNA procedure well and the practitioner satisfaction level was higher than Oxygen supplementation through a mask. The mean desaturation time was significantly shorter and the lowest saturation was significantly higher in nCPAP+Oxygen group. However, NIV may not be a good choice when patients are intolerant or have too much respiratory secretions.

Miyagi et al. [12] reported the usefulness of high flow nasal cannula (HFNC) during bronchoalveolar lavage in patients with acute respiratory failure. However Simon et al. [13] showed that NIV was better than HFN with regard to Oxygenation during bronchoscopy in patients with moderate to severe hypoxemia. HFNC could be a suitable device to deliver Oxygen during bronchoscopy when patients had mild or moderate hypoxemia. Takakuwa et al. [14] assessed the Oxygenation during EBUS-TBNA under midazolam sedation with using HFNC in their study and they suggested that HFNC was useful for preventing hypoxemia during the procedure. The mean age of the patients and the duration of procedure are similar to our study, but comorbidities and patient numbers are higher in our study. In Takakuwa et al.'s study, the pre and post $\mathrm{SpO} 2$, lowest $\mathrm{SpO} 2$, and the mean desaturation time were lower than our findings.

CO2 elevation is also a possible risk in bronchoscopy under intravenous sedation, [15]. Studies about the effects of flexible bronchoscopy have focused on the temporary alterations of gas exchange occurring during the procedure. The risk of hypoxaemia and hypercapnia caused by alveolar hypoventilation, by an increased ventilation-perfusion mismatch and metabolic demands, as reflected by increasing cardiac output and Oxygen consumption [16]. CPAP improves Oxygenation by reducing intrapulmonary shunting and work of breathing $[17,18]$. HFNC provides a low-level positive pressure $(2-8 \mathrm{cmH} 2 \mathrm{O})$. This effect could help to lung recruitment and open the upper airways similar to CPAP [19]. It has been shown that there was less carbon dioxide retention in HFNC when compared with the nasal Oxygen supply [20]. Unfortunately, we could not perform blood gas analysis and could not measure partial pressure of carbon dioxide (pCO2) levels. This is one of the limiting factors of our study. Further studies are needed on this subject. It is not clear how to define the CPAP pressure should be applied to the patients. We gave it by titration, starting with $6 \mathrm{mBar}$ and rising to maximum pressure by $14 \mathrm{mBar}$. Since the procedure is administered orally, the risk of air leakage from the mouth may reduce the effectiveness. If the nasal airflow rate exceeds the inspiratory 
flow rate, the patient can breathe spontaneously from the nasal cavity. In our study, we did not specifically exclude patients with high obstructive sleep apnea (OSA) risk. We thought that the risk of desaturation would be higher in OSA patients and CPAP would be effective especially in these patients. We can not advise the use of nCPAP during EBUS-TBNA under intravenous sedation to all patients. We suggest that the patients with high Mallampati score will benefit from nCPAP.

\section{Conclusion}

It is important to prevent desaturation in order to ensure process safety and comfort during the EBUSTBNA under intravenous sedation. Our study showed that Oxygen delivery via nCPAP was shortened the mean desaturation time during EBUS-TBNA when compared with Oxygen delivery through a simple mask at the same dose of anesthetic drugs. We advise the use of nCPAP during EBUS-TBNA under intravenous sedation to all patients. And we strongly suggest that the patients with high Mallampati score will benefit from nCPAP.

\section{References}

1. Yasufuku K, Chiyo M, Sekine Y, Chhajed PN, Shibuya K, Iizasa T, Fujisawa T. Real time endobronchial ultrasound guided transbronchial needle aspiration of mediastinal and hilar lymph nodes. Chest 2004; 126: $122-128$.

2. Lee HS, Lee GK, Lee HS, Kim MS, Lee JM, Kim HY, Nam BH, Zo JI, Hwangbo B. Real-time endobronchial ultrasound-guided transbronchial needle aspiration in mediastinal staging of non-small cell lung cancer: How many aspirations per target lymph node station? Chest 2008, 134, 368-374.

3. Clarkson K, Power CK, O'Connell F, Pathmakanthan S, Burke CM. A comparative evaluation of propofol and midazolam as sedative agents in fiberoptic bronchoscopy. Chest 1993;104: 1029-1031.

4. Dreher M, Ekkernkamp E, Storre JH, Kabitz HJ, Windisch W. Sedation during flexible bronchoscopy in patients with pre- existing respiratory failure: midazolam versus Midazolam plus Alfentanil. Respiration 2010; 79: 307-314.

5. British Thoracic Society Bronchoscopy Guidelines Committee, a Subcommittee of Standards of Care Committee of British Thoracic Society. British Thoracic Society guidelines on diagnostic flexible bronchoscopy. Thorax 2001; 56: 11-21.

6. Eastwood PR, Platt PR, Shepherd K, Maddison K, Hillman DR. Collapsibility of the upper airway at different concentrations of propofol anesthesia. Anesthesiology 2005; 103: 470-477.

7. Trachsel D, Erb TO, Frei FJ, Hammer J. Use of continuous positive airway pressure during flexible bronchoscopy in young children. European Respiratory Journal 2005 26: 773-777.

8. Chhajed PN, Reber A, Trachsel D. Frei FJ. Effect of jaw-thrust and continuous positive airway pressure on tidal breathing in deeply sedated infants. J Pediatr 2001; 138 (6): 826-830.

9. Chung F, Subramanyam R, Liao P, Sasaki E, Shapiro C, Sun Y. High STOP-Bang score indicates a high probability of obstructive sleep apnoea. Br J Anaesth. 2012; 108(5): 768-775.

10. Antonelli M, Conti G, Rocco M, Arcangeli A, Cavaliere F, Proietti R, et al. Noninvasive positivepressure ventilation vs. conventional Oxygen supplementation in hypoxemic patients undergoing diagnostic bronchoscopy. Chest 2002; 121:1149-1154.

11. Murgu SD, Pecson J, Colt HG. Bronchoscopy during noninvasive ventilation: indications and technique. Respir Care 2010; 55: 595-600.

12. Miyagi K, Haranaga S, Higa F, Tateyama M, Fujita J. Implementation of bronchoalveolar lavage using a high-flow nasal cannula in five cases of acute respiratory failure. Respir Investig 2014; 52: 310-314.

13. Simon M, Braune S, Frings D, Wiontzek AK, Klose H, Kluge S. High-flow nasal cannula Oxygen versus non-invasive ventilation in patients with acute hypoxaemic respiratory failure undergoing flexible bronchoscopy-a prospective randomised trial. Crit Care 2014; 18: 712.

14. Takakuwa O, Oguri T, Asano T, Fukuda S, Kanemitsu Y, Uemura Takehiro, Ohkubo H, Takemura M, Maeno K, Ito Y, Niimi A. Prevention of hypoxemia during endobronchial ultrasound-guided transbronchial needle aspiration: Usefulness of high-flow nasal cannula. Respiratory Investigation 2018; 56: 418-423. 
15. Foster A, Gardaz JP, Suter PM, Gemperle M. Respiratory depression by midazolam and diazepam. Anesthesiology 1980; 53: 494-497.

16. Maitre B, Jaber S, Maggiore SM, et al. Continuous positive airway pressure during fiberoptic bronchoscopy in hypoxemic patients. Am J Respir Crit Care Med 2000; 162: 1063-1067.

17. Schlobohm RM, Falltrick RT, Quan SF, Katz JA. Lung volumes, mechanics, and Oxygenation during spontaneous positive-pressure ventilation: the advantage of CPAP over EPAP. Anesthesiology 1981; 55: 416-422.

18. Katz JA, Marks JD. Inspiratory work with and without continuous positive airway pressure in patients with acute respiratory failure. Anesthesiology 1985; 63: 589-607.

19. Diab S, Fraser JF. Maintaining Oxygenation successfully with high flow nasal cannula during diagnostic bronchoscopy on a postoperative lung transplant patient in the intensive care. Case Rep Crit Care 2014.

20. Braunlich J, Seyfarth HJ, Wirtz H. Nasal high-flow versus non-invasive ventilation in stable hypercapnic COPD: a pre-liminary report. Multidiscip Respir Med 2015;10:27.

\section{Hosted file}

Table 1.pdf available at https://authorea.com/users/366198/articles/485984-the-effect-ofoxygen-application-with-ncpap-for-the-prevention-of-desaturation-during-ebus-tbna

\section{Hosted file}

Table 2.pdf available at https://authorea.com/users/366198/articles/485984-the-effect-ofoxygen-application-with-ncpap-for-the-prevention-of-desaturation-during-ebus-tbna

\section{Hosted file}

Table 3.pdf available at https://authorea.com/users/366198/articles/485984-the-effect-ofoxygen-application-with-ncpap-for-the-prevention-of-desaturation-during-ebus-tbna

\section{Hosted file}

Figure 1.pdf available at https://authorea.com/users/366198/articles/485984-the-effect-ofoxygen-application-with-ncpap-for-the-prevention-of-desaturation-during-ebus-tbna

\section{Hosted file}

Figure 2.pdf available at https://authorea.com/users/366198/articles/485984-the-effect-ofoxygen-application-with-ncpap-for-the-prevention-of-desaturation-during-ebus-tbna 